\title{
Dificultades en el resarcimiento de los daños ocasionados al patrimonio público
}

\section{Por: Sandra Jaqueline Guerrero Velandia}

\section{Resumen.}

El propósito del presente documento es identificar las dificultades que poseen los operadores jurídicos al momento de pretender la recuperación del daño ocasionado al patrimonio público por parte de quienes realizan gestión fiscal o de servidores públicos o particulares que participen, concurran, incidan o contribuyan directa 0 indirectamente en la producción del mismo.

La metodología a emplear se basa en un método mixto, teniendo en cuenta que se va a combinar un método cuantitativo como cualitativo de investigación y se tendrán como documentos de análisis, los informes de gestión y las rendiciones de cuenta de las contralorías del Departamento de Santander, respecto a los formatos F-17 Procesos de Responsabilidad Fiscal y F-18 Procesos de Jurisdicción Coactiva reportados en el SIA Misional, solicitudes de información a las contralorías, estudio de casos de manera aleatoria y encuestas a los operadores jurídicos.

Finalmente, el producto que se pretende entregar es un artículo que determine las dificultades para el resarcimiento de los daños ocasionados al patrimonio público y una aproximación a las posibles soluciones que se deben poner en marcha por los órganos de control para lograr ese objeto de la responsabilidad fiscal (Artículo 124 Decreto 403 de 2020).

Palabras claves: Proceso de responsabilidad fiscal; Control fiscal; Daño fiscal; Gestión fiscal; Resarcimiento.

\section{DIFFICULTIES IN THE COMPENSATION OF THE DAMAGES CAUSED TO THE PUBLIC PATRIMONY}

\section{Summary.}

The purpose of this document is to identify the difficulties that the legal operators have when trying to recover the damage caused to the public patrimony by those who carry out fiscal management or public servants or individuals who participate, concur, influence or contribute directly or indirectly in its production. 
Dificultades en el resarcimiento de los daños ocasionados al patrimonio público.

Página 2 de 4

The methodology to be used is based on a mixed method, bearing in mind that a quantitative method will be combined with a qualitative one of investigation and will be used as analysis documents, management reports and account statements of the comptrollers of the Department of Santander, regarding formats F-17 Fiscal Responsibility Processes and F-18 Coercive Jurisdiction Processes reported in the SIA Misional, requests for information to the comptrollers, random case studies and surveys of legal

operators.

Finally, the product that is intended to be delivered is an article that determines the difficulties for the compensation of the damages caused to the public patrimony and an approximation to the possible solutions that must be implemented by the control bodies to achieve that object of fiscal responsibility. (Article 124 Decree 403 of 2020).

Keywords: Fiscal responsibility process; Fiscal control; Fiscal damage; Fiscal management; Compensation.

\section{Ecuación de búsqueda.}

-Responsabilidad fiscal

\section{Contexto Internacional:}

- Los 10 países líderes en la investigación y producción científica del mundo en el presente tema, según el orden de clasificación son: Estados Unidos, Reino Unido, Canadá, Australia, Países Bajos, Alemania, Brasil, España, Francia e Italia. Fuente: https://www.scimagojr.com

- Colombia ocupa el puesto 39 en la producción científica mundial sobre el presente tema y el puesto tercero, a nivel regional. Fuente: https://www.scimagojr.com

- A nivel mundial las publicaciones más importantes en el tema, de acuerdo con su clasificación, son: "Administrative Science Quarterly", "Journal of Public Administration Research and Theory", "Public Administration Review", "International Public Management Journal", "Journal of Policy Analysis and Management", "Journal of European Public Policy", "Criminology \& Public Policy",

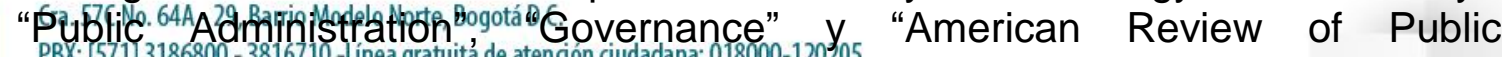

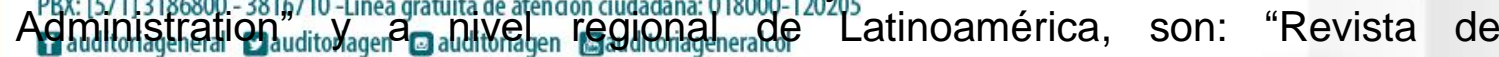
Administracaoydabliblica", "RAE Revista de Administracao de Empresas", "Revista Brasileiraiade.Politicas Publicas", "Innovar", "Reforma y Democracia" y "Gestion y Politica Publica". Fuente: https://www.scimagojr.com 
Dificultades en el resarcimiento de los daños ocasionados al patrimonio público.

Página 3 de 4

\section{Contexto regional:}

- Los países que lideran la producción académica en Latinoamérica de acuerdo con la ecuación de búsqueda y en estricto orden de clasificación son: Brasil, Colombia, México, Perú, Argentina y Ecuador. Fuente: http://www.lareferencia.info/es/

- Los cinco (5) autores que más publican en Latinoamérica de acuerdo con la ecuación de búsqueda son: Gissele Souza De Franceschi Nunes; Diego Mauricio Díaz Velásquez, Jahir Alexander Gutiérrez Ossa, Claudia Toscano Martínez y Daniel Bin. Fuente: http://www.lareferencia.info/es/

- Las universidades que más publican en Latinoamérica de acuerdo con la ecuación de búsqueda son: Universidade Federal do Rio Grande do Norte, Universidad del Rosario, Universidad de Brasilia, Colciencias y Fundação Getulio Vargas. Fuente: http://www.lareferencia.info/es/

- La tipología documental de mayor publicación en Latinoamérica de acuerdo con la ecuación de búsqueda corresponde a tesis de maestría. Fuente: http://www.lareferencia.info/es/

\section{Conclusiones.}

Se puede observar que sobre el tema específico referido a la responsabilidad fiscal a nivel de Latinoamérica, el mayor número de publicaciones corresponde a Brasil con 26, seguido de Colombia con 10. Sin embargo, al verificar las publicaciones de Brasil éstas hacen referencia a asuntos relacionados con la ley de responsabilidad fiscal existente en ese país, mientras que en Colombia se evidencia que dos de las publicaciones se refieren al tema del proceso de responsabilidad fiscal y las restantes, tocan aspectos enfocados a la responsabilidad fiscal de algunos servidores públicos o contratistas del país.

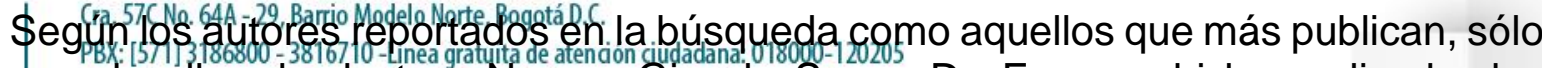

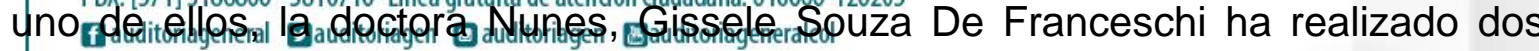
publicacionesitotine đesis de maestría y un artículo, mientras que los demás, efectúan unaws6paditpiabłl'cación que se encuentra registrada dos veces en la fuente de consulta, reportándose erróneamente dos publicaciones. 
Dificultades en el resarcimiento de los daños ocasionados al patrimonio público.

Página 4 de 4

De otra parte y en relación con el país que posee el mayor número de publicaciones sobre el tema de estudio -Brasil-, se encuentra la Universidade Federal do Rio Grande do Norte, siendo ésta la institución superior que registra el mayor número de publicaciones, con seis correspondientes a producciones de artículos. Sin embargo, resulta pertinente aclarar que la Universidad del Rosario de Colombia, toma el segundo lugar, con tres publicaciones referidas a tesis de maestría, cuya tipología documental representa el mayor número de publicaciones equivalente a 21, muy seguido de 20 artículos registrados.

Finalmente, podemos concluir que la mayoría de publicaciones sobre el tema corresponde a aquellas realizadas en Brasil, sin embargo, al analizar los contenidos de los recursos publicados, se hallan errores en la fuente de información, teniendo en cuenta que aparecen publicaciones reportadas dos veces, situación que no garantiza el $100 \%$ de la veracidad de la información analizada.

\section{Referencias.}

- Acto Legislativo 04. Diario Oficial N 51.080, Bogotá, D.C., Colombia, 18 de septiembre de 2019.

- Decreto Ley 403. Diario Oficial N 51.258, Bogotá, D.C., Colombia, 16 de marzo de 2020.

- Ley 610. Diario Oficial № 44.133, Bogotá, D.C., Colombia, 18 de agosto de 2000.

- https://www.auditoria.gov.co/atencion al ciudadano/sia. SIA Misional

- https://www.scimagojr.com/

- http://www.lareferencia.info/es/ 